\title{
Editorial
}

\section{Postoperative nausea and vomiting - when will it stop?}

W $\mathrm{E}$ can walk on the moon, and among other scientific miracles, we can perform minimally invasive, keyhole cardiac surgery with "robots". But so far we have not solved the seemingly simple problem of postoperative nausea and vomiting (PONV). It remains one of the final therapeutic challenges and has been a perpetual dilemma since the birth of our specialty. It is the legendary big, "little problem". ${ }^{1}$ Today, as we stretch the limits of ambulatory care, we are magnifying the problem. When post discharge nausea and vomiting persists after surgery, such as laparoscopic cholecystectomy, and patients cannot retain oral analgesics, their suffering is further compounded by unrelieved pain. Now we have a big, "big problem".

In the early days of ether anesthesia PONV was severe. Patients can still describe it decades later and in great detail. Ironically, it may not have been perceived as a big problem since patients and surgeons were relieved and grateful to have any anesthesia. Even profound PONV was dwarfed by the bigger problems of life-threatening morbidity and significant mortality.

Now, we have the opposite situation. New anesthetic agents have reduced the incidence and severity of PONV. Anesthesia is so safe that the major morbidity and mortality problems are rare and often due to human error. PONV is one of the most common and most important elements in morbidity. Patients assume that anesthesia is safe and refractory nausea is one of their greatest fears. ${ }^{2}$ The prevalence of PONV, coupled with high patient expectations, have made it a big problem, especially since antiemetics are not always effective.

The ideal antiemetic does not exist. ${ }^{3}$ There is no wonder drug for PONV, there aren't even drugs that come close to being called wonderful. The ideal drug would be cheap, simple, safe and effective; one drug, one dose, and always effective. There is no magic bullet for PONV. The major obstacle in solving this problem has been its multifactorial etiology. The factors involved are so numerous, so diverse and so unavoidable, that to encompass such a broad target may require a pharmacological force field rather than a single bullet. The Achilles heel of antiemetic therapy is efficacy. PONV can be alleviated, but not eliminated. This issue of poor efficacy is unique in anesthesia. Induction agents, muscle relaxants and inhalational anesthetics all produce predictable outcomes. Following the "big syringe, little syringe" routine we expect all, not just some, patients to be anesthetized. But a flip of a coin odds at $50 \%$ are considered good for prophylactic antiemetics, and rescue antiemetic therapy is like tossing out a floatation device that doesn't always float.

Since any single antiemetic drug has been shown to have limited efficacy, multimodal therapy has been proposed. In theory, the combination of several drugs in small doses should create synergistic beneficial effects, while reducing undesirable side effects. This is true when the drugs have different side effects. But many antiemetics share similar side effects - sedation. When combining antiemetics their efficacy may remain evasive while synergistic sedation becomes very evident. Luckily, drug induced drowsiness is one of the best ways to relieve PONV. Sedation makes nausea more bearable by allowing the patient to "sleep it off".

Is something better than nothing? Is an ounce of prevention worth a pound of cure? Some patients have a history of severe PONV and some surgical procedures almost guarantee PONV. So we feel justified in doing something, even if therapy is not very effective, because to do nothing seems callous, even cruel. On the other hand, it is important to consider that with every drug we give there is an additional small but real risk of 
adverse events. Antiemetics are used to treat the side effects of other drugs but they, too, have their own side effects. The goal is not to trade one set of side effects for another. Sometimes, in trying to make a bad situation better, we actually make it worse by replacing a little problem with a big problem. ${ }^{4}$ Primum non nocere.

Evidence based guidelines for cost effective therapy are urgently needed. The first step is a review of the literature which is, itself, a daunting task. There is simply too much information. But despite the vast number of studies there are huge holes of missing information. Data describing the efficacy of dimenhydrinate is virtually nonexistent, yet we use it routinely. ${ }^{5}$ Often studies have compared new and expensive drugs with placebo. The real question is the comparison of new vs old, not new $v s$ nothing. Other data is of historical value only. In many clinical trials the efficacy of prophylactic therapy was examined using anesthetic techniques specifically designed to maximize the incidence of PONV. These trials must be repeated using current techniques that minimize PONV. Another issue of fundamental importance is prophylactic $v s$ rescue therapy. Most trials involve prophylactic therapy, while rescue therapy is most clinically relevant, but least understood.

Parlow's paper ${ }^{6}$, in this issue, makes a bold attempt to define a cost effective, multimodal antiemetic protocol for routine clinical practice. The study was eloquently designed. Anesthesia and analgesia were engineered to minimize PONV. The surgical model was chosen for its well established emetic potential; female outpatients undergoing laparoscopy. Despite the combination of prophylactic and rescue therapy using droperidol, promethazine and dimenhydrinate, the incidence of PONV was unchanged. In fact, the only thing that changed were sedation scores. The desired effects were undetectable while the unwanted side effects were obvious.

PONV is a frustrating paradox; it is small stuff, but it is a big problem; with tincture of time it resolves spontaneously, but treatment with complicated prophylactic plus rescue and multimodal protocols may seem futile. PONV is a perpetual and tenacious problem that sticks to anesthesia like glue.

Since patients are preoccupied with PONV, anesthesiologists are too. It has become a topic which is fashionable, prevalent, repetitious, boring, stale, dreary, tedious, monotonous, etc., a subject we are sick of? For some readers, the mere mention of another paper on PONV will make them nauseous. So, if you feel ill at the repulsive prospect of more PONV studies - brace yourself, because further research will be required to develop new, more effective drugs, and to establish evidence based guidelines for cost effective therapy.

\section{Nausées et vomisse- ments postopératoires - quand cesseront-ils?}

Nous pouvons marcher sur la lune, et expérimenter d'autres miracles scientifiques, nous pouvons réaliser des interventions cardiaques à effraction et incision minimales grâce à des "robots". Mais, jusqu'à maintenant, nous n'avons pu résoudre le problème apparemment simple des nausées et des vomissements postopératoires (NVPO). Cette situation représente l'un des derniers défis thérapeutiques à relever, un dilemme perpétuel depuis la venue de notre spécialité. C'est le légendaire gros, "petit problème". Aujourd'hui, à mesure que nous reculons les limites des soins ambulatoires, nous amplifions ce problème. Lorsqu'après la sortie de l'hôpital, des nausées et des vomissements postopératoires persistent, comme après une cholécystectomie laparoscopique, et que les patients ne peuvent garder les analgésiques oraux, leur inconfort est aggravé par une douleur non traitée. Nous sommes alors confrontés à un gros, "gros problème".

Au début de l'usage de l'anesthésie à l'éther, les NVPO étaient sévères. Des patients peuvent encore en parler des décennies plus tard et avec force détails. Ironiquement, les NVPO n'ont peut-être pas été perçus comme un problème sérieux, heureux et soulagés qu'étaient les patients et les chirurgiens de profiter de toute forme d'anesthésie. Même les NVPO importants étaient éclipsés par les problèmes plus évidents de morbidité grave et de mortalité importante.

À présent, nous vivons la situation inverse. De nouveaux anesthésiques ont permis de réduire l'incidence et la sévérité des NVPO. L'anesthésie est si sûre que les problèmes majeurs de morbidité et de mortalité sont rares et souvent causés par des erreurs humaines. Les NVPO sont l'un des éléments les plus fréquents et les plus importants de la morbidité. Les patients considèrent que l'anesthésie est sécuritaire et craignent pardessus tout les nausées persistantes. ${ }^{2}$ La prévalence des NVPO ajoutée aux attentes élevées des patients en ont fait un problème important, surtout quand on pense que les antiémétiques ne sont pas toujours efficaces.

L'antiémétique idéal n'existe pas. ${ }^{3}$ Il n'y a pas de médicament miracle contre les NVPO, il n'y a pas de médicaments près de le devenir. Ce médicament idéal est bon marché, simple, sûr et efficace; un médicament, une dose, et toujours efficace. Il n'y a pas d'arme ma- 
gique contre les NVPO. L'obstacle principal à la résolution de ce problème réside dans son étiologie multifactorielle. Les facteurs impliqués sont si nombreux, si diversifiés et si inévitables qu'atteindre une cible aussi énorme peut exiger non plus une simple balle mais plutôt un champ d'énergie pharmacologique. La faiblesse de la thérapie antiémétique, c'est l'efficacité. On peut soulager les NVPO, mais on ne peut les éliminer. C'est un cas unique de piètre performance en anesthésie. Les agents d'induction, les myorelaxants et les anesthésiques par inhalation présentent tous des résultats prévisibles. Après une "forte ou faible injection" de routine, nous nous attendons à ce que tous les patients, et non pas quelques-uns, soient insensibilisés. Pourtant, dans le cas des antiémétiques prophylactiques, des chances de $50 \%$, comme à pile ou face, sont considérées comme bonnes, ce qui fait de la thérapie antiémétique de secours une bouée qu'on lance mais dont on n'est pas sûr de la flottaison.

Tout antiémétique utilisé seul ayant révélé son efficacité limitée, on a proposé une thérapie multimodale. Théoriquement, la combinaison de certains médicaments en petites doses devrait créer des effets bénéfiques synergiques tout en réduisant les effets secondaires indésirables. Cette affirmation s'avère quand les médicaments présentent des effets secondaires différents. Or, de nombreux antiémétiques partagent des effets secondaires similaires, dont la sédation. Lorsqu'on combine les antiémétiques, leur efficacité peut rester incomplète alors que la sédation synergique devient très évidente. Heureusement, un médicament qui provoque la somnolence représente l'un des meilleurs moyens de soulager les NVPO. La sédation rend les nausées plus supportables, puisqu'elle permet au patient de "dormir dessus".

Est-ce que quelque chose c'est mieux que rien? Un gramme de prévention vaut-il des tonnes de traitements? Certains patients ont une histoire de NVPO sévères et certaines interventions chirurgicales offrent pratiquement une garantie de NVPO. Nous nous sentons donc justifiés de faire quelque chose, même si la thérapie n'est pas très efficace, parce que ne rien faire semble insensible, voire cruel. Par contre, il faut tenir compte du fait que chaque médicament donné représente un risque additionnel faible, mais bien réel, d'effets secondaires. Les antiémétiques sont administrés pour traiter les effets secondaires d'autres médicaments, mais ils ont aussi leurs propres effets négatifs. L'objectif n'est pas de troquer un ensemble d'effets indésirables contre un autre. Parfois, en tentant d'améliorer une situation, nous l'aggravons en remplaçant un petit problème par un gros. ${ }^{4}$ Primum non nocere.
Des lignes de conduite basées sur l'évidence scientifique sont réclamées d'urgence en vue d'une thérapie rentable. La première étape pour y parvenir c'est de revoir la documentation existante, une tâche impressionnante en soi. Il y a tout simplement trop d'informations. Cependant, malgré le grand nombre d'études, il y a de grands manques d'informations. Les données qui décrivent l'efficacité du dimenhydrinate sont pratiquement inexistantes, même si nous utilisons ce médicament régulièrement. ${ }^{5}$ Les études ont souvent comparé les médicaments nouveaux et chers avec un placebo. Le véritable enjeu c'est la comparaison du nouveau vs l'ancien, et non du nouveau vs rien. D'autres données n'ont qu'une valeur historique. Dans de nombreux essais cliniques, l'efficacité de la thérapie prophylactique a été étudiée par l'emploi de techniques anesthésiques spécifiquement conçues pour maximaliser l'incidence des NVPO. Ces essais doivent se répéter en utilisant, cette fois, des techniques qui minimisent les NVPO. Un autre sujet d'importance fondamentale est la thérapie prophylactique vs la thérapie de secours. La plupart des essais concernent la prophylaxie, tandis que la thérapie de secours est plus pertinente en clinique, mais moins démontrée.

L'article de Parlow ${ }^{6}$, dans le présent numéro, ose une tentative de définition d'un protocole antiémétique rentable, multimodal pour la pratique clinique régulière. L'étude a été brillamment conçue. L'anesthésie et l'analgésie ont été organisées pour minimiser les NVPO. Le modèle chirurgical a été choisi pour son potentiel émétique bien connu; les patientes subissaient une laparoscopie. Malgré la combinaison de thérapies prophylactiques et de secours à base de dropéridol, de prométhazine et de dimenhydrinate, l'incidence de NVPO est restée la même. De fait, la seule chose qui a changé c'est le score de sédation. Les effets désirés ont été indétectables tandis que les effets secondaires indésirables ont été évidents.

Les NVPO présentent un paradoxe frustrant; ce n'est pas grand-chose, mais c'est un gros problème; en un rien de temps, cela se résorbe spontanément et le traitement fait de protocoles multimodaux prophylactiques et de secours compliqués peuvent sembler futiles. Les NVPO sont un problème perpétuel et tenace qui s'attache à l'anesthésie comme une colle.

Les patients étant préoccupés par les NVPO, les anesthésiologistes le sont également. C'est devenu un sujet à la mode, prédominant, répétitif, rasant, lassant, ennuyeux, fastidicux, monotone, etc., un sujet dont on a marre! Pour certains, la seule mention d'un autre article sur les NVPO leur donne la nausée. Donc, si vous vous sentez malade à l'idée répulsive d'autres études sur les NVPO, préparez-vous, car d'autres 
études sont nécessaires pour développer des médicaments nouveaux et efficaces et pour établir des lignes de conduite, basées sur des évidences scientifiques, de rentabilité thérapeutique.

\section{References}

1 Kapur PA. The big "little problem". (Editorial) Anesth Analg 1991; 73: 243-5.

2 Orkin F. What do patients want? - Preferences for immediate postoperative recovery. Anesth Analg 1992; 74: S225.

3 Watcha MF, White PF. Postoperative nausea and vomiting. Its etiology, treatment, and prevention. Anesthesiology 1992; 77: 162-84.

4 Ross $A K$, Ferrero-Conover $D$. Anaphylactoid reaction due to the administration of ondansetron in a pediatric neurosurgical patient. Anesth Analg 1998; 87: 779-80.

5 Vener DF, Carr AS, Sikish N, Bissonette B, Lerman J. Dimenhydrinate decreases vomiting after strabismus surgery in children. Anesth Analg 1996; 82: 728-31.

6 Parlow JL, Meikle AT, Van Vlymen J. Post discharge nausea and vomiting after ambulatory laparoscopy is not reduced by promethazine prophylaxis. Can J Anesth 1999; 46: 719-24. 